\title{
Nanoceria and Its Biomedical Relevance
}

\author{
Jyotsna Kailashiya ${ }^{1}$ Debabrata Dash ${ }^{1}$ \\ ${ }^{1}$ Department of Biochemistry, Institute of Medical Sciences, \\ Banaras Hindu University, Varanasi, Uttar Pradesh, India
}

\begin{abstract}
Address for correspondence Debabrata Dash, MD, PhD, DSC, FAMS, FNA, FASc, Department of Biochemistry, Institute of Medical Sciences, Banaras Hindu University, Varanasi 221005, Uttar Pradesh, India (e-mail: ddash.biochem@gmail.com).
\end{abstract}

\author{
Abstract \\ Keywords \\ - cerium oxide \\ nanoparticles \\ - antioxidant \\ - reactive oxygen \\ species \\ - inflammatory diseases
}

Nanoceria is a nanosized particle preparation of cerium oxide. It shows mixture of cerium in the $3+$ and $4+$ states on the nanoparticle surface, giving it interesting redox properties. Nanoceria shows effective biological antioxidant properties, which makes it a great candidate for biomedical applications. Many studies have shown promising results on therapeutic potential of nanoceria in diseases like cancer, diabetes, atherosclerosis, and neurodegenerative diseases. Meanwhile, other studies explored biodistribution and toxicity of nanoceria. This review article describes nanoceria, its relevant biomedical applications, and adverse effects, based on previously reported studies.

\section{Introduction}

Cerium is a rare earth metal having atomic number 58 , belonging to lanthanide series. Unlike most rare earth metals, cerium can exist in both $3+$ and $4+$ states and thus, oxides of cerium exist as both $\mathrm{CeO}_{2}$ and $\mathrm{Ce}_{2} \mathrm{O} .^{1,2}$ Cerium oxide nanoparticles (nanoceria) show mixture of erium in the $3+$ and $4+$ states on their surface, giving them interesting redox properties. Nanoceria is being widely used in fields of chemicals, cosmetics, mechanical polishing/planarization, corrosion protection, solar cells, fuel oxidation catalysis, and automotive exhaust treatment. ${ }^{1,2}$ Other than these applications, nanoceria also displays many biorelevant activities-mimicking superoxide dismutase (SOD), catalase, peroxidase, oxidase, and phosphatase, and scavenging hydroxyl radicals, nitric oxide radicals, and peroxynitrite. ${ }^{1}$ These biorelevant activities of nanoceria can be used in pharmacological agents, drug delivery, and bioscaffolding. ${ }^{1}$

\section{Synthesis of Nanoceria}

Numerous techniques, such as green synthesis, hydrothermal, solvothermal, aqueous precipitation, reversed micelles, thermal decomposition, and flame spray methods have been reported to synthesize nanoceria, while maintaining control of its size and properties. ${ }^{1-3}$ The synthesized nanoceria can be bare or wrapped with a coating of protective substances that can be hydrophilic or hydrophobic. ${ }^{3}$ Naked nanoceria has poor water solubility, and thus limited biological applications. Polymer coating of nanoceria enhances its stability, biocompatibility, and water solubility. ${ }^{2}$ For biological use, biocompatible nanoceria has been systematically synthesized in pure water or with coating/functionalization of polyethylene glycol, dextran, polyacrylic acid, cyclodextrin, glucose, and so on. ${ }^{3,4}$ Methods of nanoceria preparation or synthesis are important because they determine the solubility, size, surface condition, charge, structural arrangement, and morphology of nanoparticles, thus affecting their properties, including catalytic activities. ${ }^{3}$

\section{Mechanism of Action}

The basis for activities of nanoceria is the thermodynamic efficiency of redox-cycling between $3+$ and $4+$ states on their surface and their unique ability to take up and release oxygen. ${ }^{1}$ Nanoceria could have a dual role as an oxidation catalyst and reduction catalyst, depending on the reaction conditions and surrounding microenvironment. The cerium atom on surface of nanoceria has the ability to easily and drastically adjust its electronic configuration to fit its immediate environment, and thus contributing to redox and antioxidant properties. ${ }^{3}$ The $\mathrm{Ce}^{3+} / \mathrm{Ce}^{4+}$ valence switching capacity of nanoceria makes it an SOD mimic. ${ }^{3}$ Nanoceria could also act as catalase mimic in a redox-state-dependent manner, and higher levels of cerium in the +4 state exhibit higher activity. ${ }^{3}$ Nanoceria can also show pro-oxidant properties at lower $\mathrm{pH}$ and high concentrations, and it has shown potential toxicity based on synthesis method, concentration, and exposure time. ${ }^{5}$ 


\section{Biomedical Applications}

Increased oxidative stress has been found to be associated with many neurodegenerative and chronic inflammatory diseases like Alzheimer's, Parkinsonism, Rheumatoid Arthritis (RA), Ischemic stroke, and diabetes etc. Due to its antioxidant properties, nanoceria has been studied extensively for treatment of these disorders. ${ }^{3,6}$ Nanoceria shows neuroprotective effects by protecting against free radical/ reactive oxygen species (ROS) mediated injuries. Nanoceria administration in rat model of Parkinsonism (6-OHDA-induced) resulted in partial neuroprotection against disturbances in motor performance, partially through their antioxidant and antiapoptotic effects. ${ }^{\text {? }}$ Many studies have also confirmed antibacterial activity of nanoceria against Pseudomonas aeruginosa, E. coli, B. subtilis, Shewanella oneidensis and Pseudokirchneriella supcapitata. ${ }^{2}$

Diabetes is also believed to be associated with oxidative stress and beneficial effects of nanoceria have been demonstrated in diabetic rats. ${ }^{8}$ In another study, nanoceria treatment significantly reduced glucose levels and diabetogenesis in streptozocin induced diabetic Swiss mice. In addition, cytokines (IL-6 and TNF- $\alpha$ and p65-NF- $\mathrm{BB}$ ) expression were diminished by nanoceria treatment, whereas the nuclear factor erythroid 2-related factor 2 ( $\mathrm{Nrf} 2$ ) expression was enhanced, indicating the role of modulation of NF- $\mathrm{kB} / \mathrm{Nrf} 2$ pathway. Nanoceria also exhibited promising superoxide dismutase 1 mimetic and antiapoptotic activity in these diabetic mice. ${ }^{9}$

Nanoceria has also been shown to reduce retinal degeneration by reducing ROS generation in rat retinal cells. ${ }^{10}$ The alginate-gelatin injectable hydrogel loaded with oligo-chitosan coated nanoceria showed good biocompatibility and high potential in protecting cells from apoptosis, angiogenesis, and production of proinfammatory cytokines in age related macular degeneration cellular models. ${ }^{11}$

Increased ROS production in synovium causes chronic inflammation and contributes to rheumatoid arthritis. Nanoceria and manganese ferrite nanoparticles anchored to mesoporus silica nanoparticles showed synergistic effects by scavenging ROS and promoting recruitment of anti-inflammatory macrophages in joints of rat RA model. ${ }^{12}$ Nanoceria also showed therapeutic potential by preventing valvular calcification mediated by ROS related damage,,$^{13}$ cardio-protective effects by preventing myocardial remodelling, ${ }^{14}$ and attenuating ischemia reperfusion induced hepatic injury in rats. ${ }^{15}$

Antioxidant and anti-inflammatory properties of nanoceria were found beneficial in cisplatin induced nephrotoxicity. As nanoceria ameliorated oxidative stress by showing a reduction in levels of malondialdehyde, increased levels of endogenous antioxidants, reduced glutathione and catalase, and decreased levels of proinflammatory cytokines. ${ }^{16}$ In vivo studies in mice with induced liver toxicity (by carbon tetrachloride [CCl4]) showed that nanoceria administered mice exhibit findings similar to mice treated with $\mathrm{N}$-acetyl cystine (NAC), a common therapeutic to reduce oxidative stress. ${ }^{17}$

Nanoceria has also shown potential for treatment of smoking-related diseases by exhibiting ability to protect against cigarette smoke extract (CSE)-induced oxidative stress and inflammation in cultured rat H9c2 cardiomyocytes. Results indicated that nanoceria can inhibit CSE-induced cell damage via inhibition of ROS generation, NF- $\alpha$ B activation, inflammatory gene expression, and antioxidant depletion. ${ }^{18}$ Another study showed anti-inflammatory and antioxidant effects of nanoceria in both healthy rats, and rats with pneumonia. ${ }^{19}$

Many studies have shown that nanoceria is toxic to cancer cells. ${ }^{3}$ Nanoceria induces ROS mediated damage, lipid peroxidation, apoptosis, and membrane leakage in cancer cells, but not in healthy/normal cells. ${ }^{3}$ This has been attributed to cancer cells having a more acidic cytosolic $\mathrm{pH}$ than normal cells because of higher glycolysis and lactate production. ${ }^{3}$ Although, results from few studies are conflicting. Nanoceria treatment to human monocytic leukemia cells (THP-1) resulted in reduction of ROS but cytotoxicity was not observed. ${ }^{20}$ A study in broncho-alveolar carcinoma-derived A549 cells showed that uptake of the nanoceria resulted in slight change of the cell cycles, i.e., more cells stayed in the G1 phase but the cell viability was not significantly altered. ${ }^{21}$

When functionalized with anticancer molecules, nanoceria demonstrate synergistic toxic effects in cancer cells., ${ }^{3,22,23}$ Protective effects of nanoceria on healthy cells, while killing glioma cancer cells have been reported. ${ }^{24}$ Nanoceria abolished toxic effects of anticancer drug (doxorubicin) on human dermal fibroblasts. ${ }^{25}$

The multi-enzyme-like properties of nanoceria have been successfully used for biological detection and analysis, e.g., colorimetric immunoassays, enzyme-linked immunosorbent assay (ELISA), and biosensors etc., ${ }^{3,26-28}$

\section{Biodistribution}

In vivo analysis of the biological distribution of nanoceria administered to mice perorally (PO), intravenously (IV), or intraperitoneally (IP) showed that the IV and IP administration leads to most extensive and cumulative nano-deposition while PO administration led to excretion of greater than $95 \%$ nanoceria within 24 hours. Organ deposition for IV and IP mice was highest in the spleen followed by the liver, lungs, and kidneys. Nanoceria was excreted through feces all administration routes. ${ }^{17}$ Study in C. elegans showed that positively charged nanoceria is significantly more toxic and accumulated to a greater extent than the neutral and negatively charged nanoceria. ${ }^{29}$

\section{Toxicity and Adverse Effects}

Cerium is not found in the human body and there are no known clearance mechanisms for it. This implies that exposure to cerium would lead to systemic toxicity. ${ }^{1}$ Nanoceria is successfully up taken by cells in both normal and diseased states through multiple routes. In most cases of in vitro intracellular assays, nanoceria was reported to exhibit positive effects (such as scavenging ROS) and was regarded as a promising biomaterial for biomedical applications. However, several reports suggested that the uptake of nanoceria could induce oxidative stress and DNA damage, apoptosis, dephosphorylation of various substrates, aberrant cell signaling, and alterations in the transcriptional and posttranslational levels. ${ }^{3,30-32}$ 
In vivo analyses of the biological effect of different sizes of nanoceria have been performed by per-oral, intravenous or intraperitoneal administration to laboratory animals.,33 There are few studies showing that exposure of animals to nanoceria resulted in significant lung responses, including lung inflammation, cytotoxicity, lung injury, alveolar macrophage functional changes, induction of phospholipidosis, and release of proinflammatory and fibrotic cytokines. Cerium is also linked to fibrosis of the heart, and nanoceria was shown to induce myocardial fibroblast proliferation and collagen deposition in rats.

Due to the extensive use, nanoceria is getting released to the environment and humans are getting exposed, mostly via inhalation. ${ }^{2}$ Nanoceria is used as a diesel fuel catalyst, and thus can be emitted into air, resulting in exposure to humans by inhalation. A recent study investigated the acute (24 hours) effect of intratracheal (IT) instillation of nanoceria on pulmonary and systemic inflammation, oxidative stress and thrombosis in mice. Results showed that acute pulmonary exposure to nanoceria induced pulmonary and systemic inflammation, oxidative stress, and thrombosis in vivo. ${ }^{34}$ Nanoceria administration has shown to result in elevated WBC counts after IV and IP administration in mice, ${ }^{17}$ and hepatic injury with oxidative stress in rats after single vascular infusion. ${ }^{35}$

Above data necessitates careful optimization of applications and synthesis parameters to generate nontoxic nanoceria that are based on the treatment strategy being used, and further exploration of biochemical effects of nanoceria. ${ }^{1,3}$

Conflict of Interest

None declared.

\section{References}

1 Dhall A, Self W. Cerium oxide nanoparticles: a brief review of their synthesis methods and biomedical applications. Antioxidants 2018;7(8):E97

2 Rajeshkumar S, Naik P. Synthesis and biomedical applications of Cerium oxide nanoparticles-A Review. Biotechnol Rep (Amst) 2017;17:1-5

3 Xu C, Qu X., Cerium oxide nanoparticle: a remarkably versatile rare earth nanomaterial for biological applications. NPG Asia Materials 2014;6(3):e90

4 CaputoF,MameliM,SienkiewiczA, etal.Anovel syntheticapproach of cerium oxide nanoparticles with improved biomedical activity. Sci Rep 2017;7(1). Doi: 10.1038/s41598-017-04098-6

5 Yokel RA, Hussain S, Garantziotis S, Demokritou P, Castranova V, Cassee FR. The Yin: An adverse health perspective of nanoceria: uptake, distribution, accumulation, and mechanisms of its toxicity. Environ Sci Nano 2014;1(5):406-428

6 Naz S, Beach J, Heckert B, et al. Cerium oxide nanoparticles: a 'radical' approach to neurodegenerative disease treatment. Nanomedicine (Lond) 2017;12(5):545-553

7 Hegazy MAE, Maklad HM, Elmonsif DAA, Elnozhy FY, Alqubiea MA, Alenezi FA. The possible role of cerium oxide $\left(\mathrm{CeO}_{2}\right)$ nanoparticles in prevention of neurobehavioral and neurochemical changes in 6-hydroxydopamineinduced parkinsonian disease. Alexandria Journal of Medicine 2017;53(4):351-360

8 Pourkhalili N, Hosseini A, Nili-Ahmadabadi A, et al. Biochemical and cellular evidence of the benefit of a combination of cerium oxide nanoparticles and selenium to diabetic rats. World J Diabetes 2011;2(11):204-210

9 Khurana A, Tekula S, Godugu C. Nanoceria suppresses multiple low doses of streptozotocin-induced Type 1 diabetes by inhibition of Nrf2/NF- $\kappa$ B pathway and reduction of apoptosis. Nanomedicine (Lond) 2018;13(15):1905-1922

10 Chen J, Patil S, Seal S, McGinnis JF. Rare earth nanoparticles prevent retinal degeneration induced by intracellular peroxides. Nat Nanotechnol 2006;1(2):142-150

11 Wang K, Mitra RN, Zheng M, Han Z. Nanoceria-loaded injectable hydrogels for potential age-related macular degeneration treatment. J Biomed Mater Res A 2018;106(11):2795-2804

12 Kim J, Kim HY, Song SY, et al. Synergistic oxygen generation and reactive oxygen species scavenging by manganese ferrite/ceria co-decorated nanoparticles for rheumatoid arthritis treatment. ACS Nano 2019;13(3):3206-3217

13 Xue Y, St Hilaire C, Hortells L, Phillippi JA, Sant V, Sant S. Shape-specific nanoceria mitigate oxidative stress-induced calcification in primary human valvular interstitial cell culture. Cell Mol Bioeng 2017;10(5):483-500

14 Kumari P, Saifi MA, Khurana A, Godugu C. Cardioprotective effects of nanoceria in a murine model of cardiac remodeling. J Trace Elem Med Biol 2018;50:198-208

15 Manne N, Arvapalli R, Graffeo VA, et al. Prophylactic treatment with cerium oxide nanoparticles attenuate hepatic ischemia reperfusion injury in sprague dawley rats. Cell Physiol Biochem 2017;42(5):1837-1846

16 Saifi MA, Sangomla S, Khurana A, Godugu C. Protective effect of nanoceria on cisplatin-induced nephrotoxicity by amelioration of oxidative stress and pro-inflammatory mechanisms. Biol Trace Elem Res 2019;189(1):145-156

17 Hirst SM, Karakoti A, Singh S, et al. Bio-distribution and in vivo antioxidant effects of cerium oxide nanoparticles in mice. Environ Toxicol 2013;28(2):107-118

18 Niu J, Wang K, Kolattukudy PE. Cerium oxide nanoparticles inhibit oxidative stress and nuclear factor $-\kappa \mathrm{B}$ activation in H9c2 cardiomyocytes exposed to cigarette smoke extract. J Pharmacol Exp Ther 2011;338(1):53-61

19 Serebrovska Z, Swanson RJ, Portnichenko V, et al. Antiinflammatory and antioxidant effect of cerium dioxide nanoparticles immobilized on the surface of silica nanoparticles in rat experimental pneumonia. Biomed Pharmacother 2017;92:69-77

20 Patel P, Kansara K, Singh R, et al. Cellular internalization and antioxidant activity of cerium oxide nanoparticles in human monocytic leukemia cells. Intl J Nanomed 2018;13(T-NANO 2014 Abstracts):39-41

21 Zhou X, Wang B, Chen Y, Mao Z, Gao C. Uptake of cerium oxide nanoparticles and their influences on functions of A549 cells. J Nanosci Nanotechnol 2013;13(1):204-215

22 Xu C, Lin Y, Wang J, et al. Nanoceria-triggered synergetic drug release based on $\mathrm{CeO}(2)$-capped mesoporous silica host-guest interactions and switchable enzymatic activity and cellular effects of $\mathrm{CeO}(2)$ Adv Healthc Mater 2013;2(12):1591-1599

23 Sack M, Alili L, Karaman E, et al. Combination of conventional chemotherapeutics with redox-active cerium oxide nanoparticles-a novel aspect in cancer therapy. Mol Cancer Ther 2014;13(7):1740-1749

24 Sack-Zschauer M, Bader S, Brenneisen P, Cerium oxide nanoparticles as novel tool in glioma treatment: an. Vitro study J Nanomed Nanotechnol 2017;8(6) 474

25 Minotti G, Menna P, Salvatorelli E, Cairo G, Gianni L. Anthracyclines: molecular advances and pharmacologic developments in antitumor activity and cardiotoxicity. Pharmacol Rev 2004;56(2):185-229

26 Song HP, Jang JY, Bae SH, Choi SB, Yu BJ, Kim MI. Convenient colorimetric detection of thrombin via aptamer-mediated 
inhibition and restoration of the oxidase activity of nanoceria. J Nanosci Nanotechnol 2018;18(9):6570-6574

27 Ni P, Xie J, Chen C, et al. Spectrophotometric determination of the activity of alkaline phosphatase and detection of its inhibitors by exploiting the pyrophosphate-accelerated oxidase-like activity of nanoceria. Mikrochim Acta 2019;186(5):320

28 Liao H, Liu Y, Chen M, Wang M, Yuan H, Hu L. A colorimetric heparin assay based on the inhibition of the oxidase mimicking activity of cerium oxide nanoparticles. Mikrochim Acta 2019;186(5):274

29 Collin B, Oostveen E, Tsyusko OV, Unrine JM. Influence of natural organic matter and surface charge on the toxicity and bioaccumulation of functionalized ceria nanoparticles in Caenorhabditis elegans. Environ Sci Technol 2014;48(2):1280-1289

30 Könen-Adıgüzel S, Ergene S. In vitro evaluation of the genotoxicity of $\mathrm{CeO}_{2}$ nanoparticles in human peripheral blood lymphocytes using cytokinesis-block micronucleus test, comet assay, and gamma H2AX. Toxicol Ind Health 2018;34(5):293-300
31 Eskandari N, Nejadi Babadaei MM, Nikpur S, et al. Biophysical, docking, and cellular studies on the effects of cerium oxide nanoparticles on blood components: in vitro. Int J Nanomedicine 2018;13:4575-4589

32 Hussain S, Al-Nsour F, Rice AB, et al. Cerium dioxide nanoparticles induce apoptosis and autophagy in human peripheral blood monocytes. ACS Nano 2012;6(7):5820-5829

33 Yokel RA, Tseng MT, Dan M, et al. Biodistribution and biopersistence of ceria engineered nanomaterials: size dependence. Nanomedicine (Lond) 2013;9(3):398-407

34 Nemmar A, Al-Salam S, Beegam S, Yuvaraju P, Ali BH. The acute pulmonary and thrombotic effects of cerium oxide nanoparticles after intratracheal instillation in mice. Int J Nanomedicine 2017;12:2913-2922

35 Tseng MT, Lu X, Duan X, et al. Alteration of hepatic structure and oxidative stress induced by intravenous nanoceria. Toxicol Appl Pharmacol 2012;260(2):173-182 